\title{
Depth cues, rather than perceived depth, govern vergence
}

\author{
D. A. Wismeijer $\cdot$ R. van Ee $\cdot$ C. J. Erkelens
}

Received: 5 May 2007 / Accepted: 21 July 2007 / Published online: 24 August 2007

(C) Springer-Verlag 2007

\begin{abstract}
We studied the influence of perceived surface orientation on vergence accompanying a saccade while viewing an ambiguous stimulus. We used the slant rivalry stimulus, in which perspective foreshortening and disparity specified opposite surface orientations. This rivalrous configuration induces alternations of perceived surface orientation, while the slant cues remain constant. Subjects were able to voluntarily control their perceptual state while viewing the ambiguous stimulus. They were asked to make a saccade across the perceived slanted surface. Our data show that vergence responses closely approximated the vergence response predicted by the disparity cue, irrespective of voluntarily controlled perceived orientation. However, comparing the data obtained while viewing the ambiguous stimulus with data from an unambiguous stimulus condition (when disparity and perspective specified similar surface orientations) revealed an effect of perspective cues on vergence. Collectively our results show that depth cues rather than perceived depth govern vergence.
\end{abstract}

Keywords Depth perception - Vergence - Disparity · Ambiguous

D. A. Wismeijer $(\varangle) \cdot$ R. van Ee · C. J. Erkelens Helmholtz Institute, Department Physics of Man, Utrecht University, Princetonplein 5, 3584 CC Utrecht, The Netherlands e-mail: D.A.Wismeijer@phys.uu.nl

C. J. Erkelens

e-mail: C.J.Erkelens@phys.uu.nl

\section{Introduction}

In vision, binocular fusion is facilitated by vergence. Various different types of horizontal vergence have been distinguished based on inputs used by the vergence system, e.g. binocular disparity and accommodation. One of those, fusional vergence, uses binocular disparity as input. And another, proximal vergence, is associated with "knowledge of nearness" (Howard, 2002). Recently, the influence of depth perception on vergence has regained interest, because of the possibility to probe perception through vergence. In several studies, various depth cues were used to induce depth and the influence of perception on vergence was studied both without and with binocular disparity present. In this study, we investigate whether depth perception per se contributes to vergence.

Enright (1987b) measured vergence movements corresponding to the depth relations implied by perspective foreshortening, under monocular viewing conditions. $\mathrm{He}$ concluded that the perceived depth suggested by perspective foreshortening could elicit vergence. Ringach et al. (1996) used the kinetic depth effect to induce perception of depth. They measured vergence, also under monocular viewing conditions, and found that vergence corresponded to depth perceived by the subject. This led them to conclude that perceived depth can elicit vergence.

More recently, several studies investigated the influence of perceived depth on vergence in the presence of disparity, i.e. under binocular viewing conditions. Sheliga and Miles (2003) used Ogle's induced effect in which vertical disparities give rise to depth. The authors reported that depending on the condition, a maximum of $41 \%$ of the vergence could be attributed to perceived depth. Both et al. (2003) used Werner's illusion to induce depth. They reported slight vergence responses corresponding to perceived depth. These 
results led to the conclusion that perceived depth contributed to the vergence responses. ${ }^{1}$

However, in all aforementioned studies, depth perception was correlated with stimulus properties, i.e. the depth cues giving rise to the perceived depth. Therefore, the distinction between an effect on vergence caused by perception and an effect due to depth cues could not be made. Thus, it may be possible that signals related to the depth cues contributed to vergence rather than perceived depth itself.

To investigate whether perceived depth itself is sufficient to influence vergence, a condition is required in which depth perception is not correlated with both monocular and binocular cues that give rise to the perceived depth (Allison et al. 1998; Gillam 1968; Gillam and Ryan 1992; Gillam and Cook 2001). The slant rivalry stimulus offers the possibility to dissociate vergence effects due to depth cues from those caused by perceived depth (van Ee et al. 2002). To create a slant rivalry stimulus, perspective foreshortening and binocular disparity are used to define slanted surfaces. If the cues specify opposite slant orientations, subjects report alternations between perceiving a slanted rectangle and perceiving an oppositely slanted trapezoid (van Ee et al. 2002). Thus, perceived slant alternates, whereas the depth cues remain constant.

Using these slant stimuli, the contribution to vergence predicted by perception is different from that predicted by the depth cues. If perceived depth is sufficient to influence vergence, a difference in vergence should be observed while alternations occur between the two possibly perceived surface slants. However, if perceived slant does not influence vergence, vergence should remain stable regardless of the perceived surface slant orientation.

To study the effect of perceived surface slant on vergence, we analyzed saccades made under various stimulus and perceptual conditions. Binocular saccades usually contain a conjugate component (version) and a disjunctive component (vergence) (Erkelens et al. 1989). Both components are preprogrammed, thus if perceived depth influences vergence, the disjunctive component should be related to the depth direction specified by the perceived slant. Hence, measured vergence at saccade offset can give insight into the input that drives the vergence system.

\footnotetext{
${ }^{1}$ After we finished this work another study was published that reported on vergence changes while observers fixated a hollow mask under different viewing orientations (Hoffmann and Sebald 2007). However, from this paper it is not clear how the (low-resolution) eye posture measurements of individual subjects, as well as their current perceptual state, contribute to the conclusion.
}

\section{Methods}

\section{Experimental setup}

Stimuli were displayed using a conventional Wheatstone stereoscope consisting of two TFT displays $\left(20^{\prime \prime}\right.$ LaCie Photon20Vision II, $1,600 \times 1,200,75 \mathrm{~Hz}$ ) and two small mirrors, see Fig. 1. The mirrors were slanted about the vertical axis at an angle of $45^{\circ}$ with respect to the display. The virtual intersection point of the orthogonal mirrors was aligned with the center of the displays. Subjects were seated close to the mirrors so that the left eye could not see the right mirror and vice versa. The straight-ahead viewing distance (eye-mirror-display) was $57 \mathrm{~cm}$. Subjects' heads were fixated using a bite-board.

Eye movements were measured using the head-mounted Eyelink I system at $250 \mathrm{~Hz}$. The cameras were positioned beneath the mirrors. The whole setup and experimental room were painted black matte and the room was darkened.

\section{Stimuli}

A stimulus consisted of two images displayed, one on each of the displays. The displayed images were generated using custom Open GL based software. The images used were trapezoidal or rectangular shapes composed of lines and a sparse random dot texture, as shown in Fig. 2. The images were surrounded by a fronto-parallel sparse random dot background (not shown).

Perspective foreshortening of both images corresponded with a 2D projection (cyclopean viewpoint) of a rectangular surface slanted $( \pm) 70^{\circ}$ about the vertical axis. A horizontal (binocular) disparity gradient defined a $(\mp) 50^{\circ}$ slant about

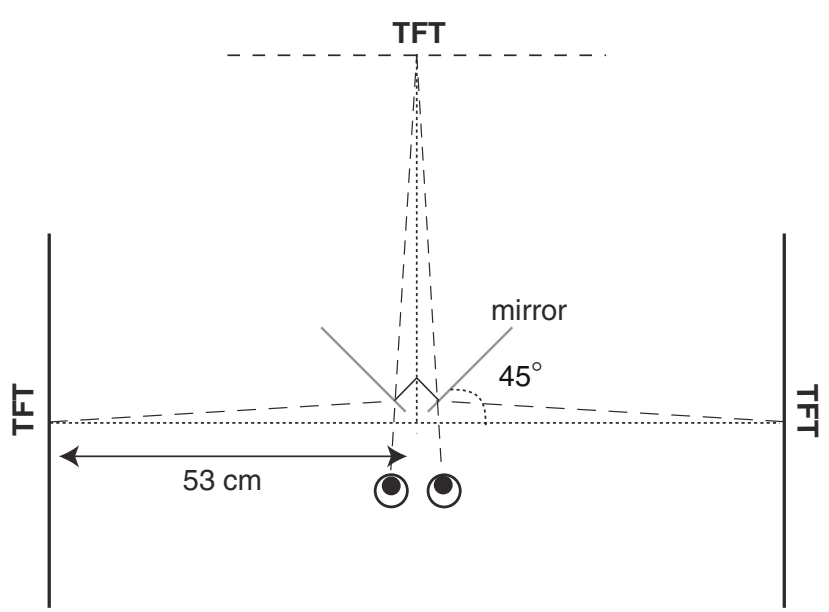

Fig. 1 Wheatstone stereoscope. Subjects viewed one TFT display with the corresponding eye via one of the mirrors. The viewing distance (eye-mirror-display) was $57 \mathrm{~cm}$. Note that the subjects were in reality much closer to the mirrors than depicted here and that there was no crossover, i.e. each eye could only see via one mirror 


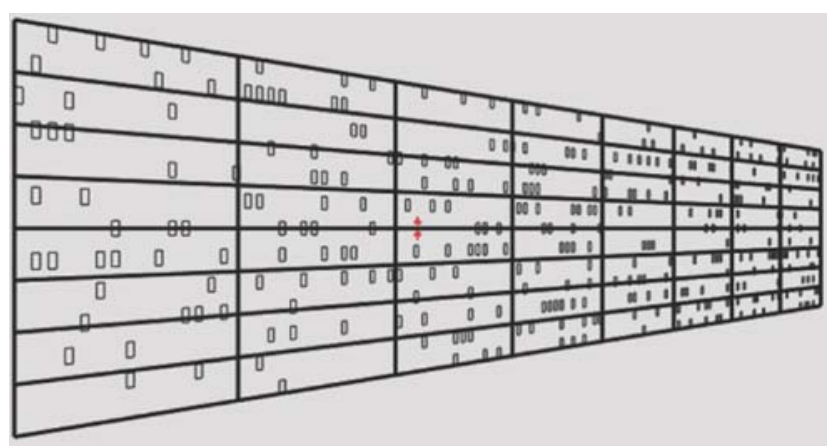

Fig. 2 An image as shown on one display. Perspective foreshortening indicates a slant of $70^{\circ}$. The disparity gradient was produced by horizontally scaling the two eyes' half images. The red fixation cross is positioned in the center of the stimulus

the vertical axis, which was produced by scaling the two eyes' half images horizontally. After scaling, the horizontal angular width of the stimulus was $20^{\circ}$ for all stimuli independent of the imposed slant angles, see Fig. 3a. The same figure also shows that a counter clockwise rotation (CCW) about the vertical axis was defined as a positive slant angle and a clockwise rotation (CW) as a negative slant angle.

Stimulus parameters could define any of the following possibilities: (1) a pair of trapezoids in which the signs of slant defined by perspective foreshortening $(P)$ and disparity $(D)$ were equal [unambiguous stimulus, e.g. $P=70^{\circ}$ and $D=50^{\circ}$, see Fig. $\left.3 \mathrm{~b}(1)\right]$ or (2) a pair of trapezoids in which
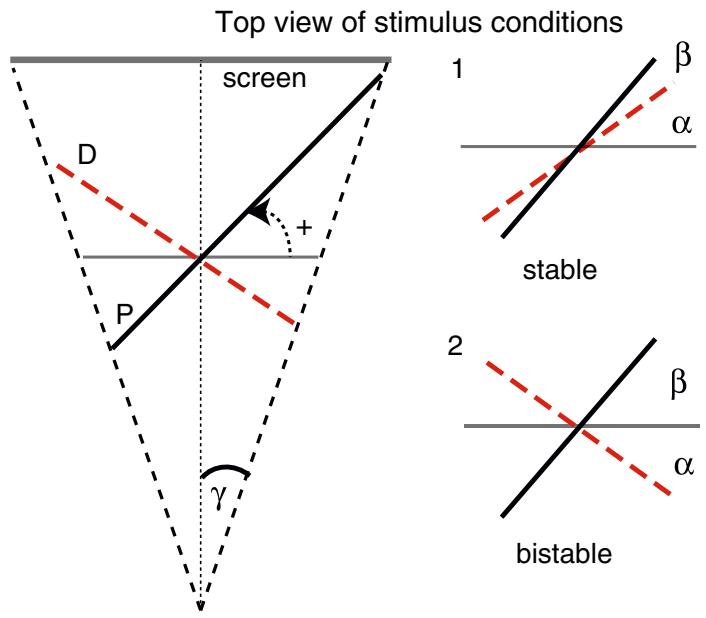

stable

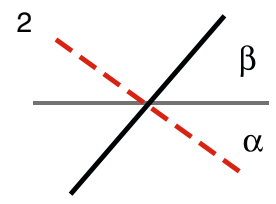

bistable

Fig. 3 a The subtended angle from center to left or right side of the stimulus was $10^{\circ}(\gamma)$ irrespective of the imposed surface slants defined by disparity $(D)$ and perspective $(P)$. A counter clockwise rotation of the surface about the vertical axis is defined as a positive surface slant angle (+). b Examples of different stimulus conditions. The surface slant angle as defined by disparity was $\pm 50^{\circ}(\alpha)$ and the perspective defined surface slant angle $(\beta)$ was $\pm 70^{\circ}$. In the unambiguous stimulus, disparity and perspective defined the same surface orientation yielding a stable perception of surface slant (1). Whereas in the ambiguous stimulus condition (2), the orientations of the defined surfaces were opposite, giving a bistable perception of surface slant

the signs of slant defined by perspective foreshortening and disparity were opposite [ambiguous stimulus, e.g. $P=70^{\circ}$ and $D=-50^{\circ}$, see Fig. $3 \mathrm{~b}(2)$ ]. These physical parameters led to the following perceived slanted surfaces: ad (1) a slanted rectangle (stable slant) or ad (2) alternations of a slanted rectangle and an oppositely slanted trapezoid (bistable slant). Note that the slant angle defined by the monocular cues is larger than the angle defined by the binocular cues, to produce a sufficient degree of bistability for all subjects. The disparity gradient could not be diminished, as this would deteriorate the signal-to-noise ratio of vergence (see Sect. 2.5 as well).

Furthermore, a standing disparity was added to all stimuli, which made all surfaces appear to be positioned in front of the display. A fixation cross was presented in the center of the stimulus.

\section{Procedure and tasks}

Experimental trials consisted of a sequence of five different displayed items. Subjects were first presented with a fixation dot (used for offline drift correction) in the center of the display at display depth for $1.5 \mathrm{~s}$ (1). This dot was replaced by a fixation cross at the location and depth corresponding to the center of the stimulus (2). After $1.5 \mathrm{~s}$, the stimulus images were added (3). A beep was sounded $2.0 \mathrm{~s}$ after stimulus onset. A monocular arrow appeared $2 \mathrm{~s}$ thereafter in the left eye's image (4). The screen was blanked after $4 \mathrm{~s}$ (5).

During the trial, subjects were instructed to fixate the cross. When the monocular arrow had appeared, subjects were instructed to make one single saccade towards the side of the surface indicated by the arrow. The arrow always pointed towards the near side of the slanted surface defined by disparity. By doing this the vergence response (disjunctive part of saccade) was maximized, because some subjects showed considerably less vergence change when saccades were made towards the uncrossed disparity side of the stimulus. This is in agreement with the effect of version-vergence ratio on the disjunctive part of the saccade as described by Enright (1984).

Subjects had to report their percept prior to saccade onset. They were asked to report the perceived nearest side of the stimulus after the beep using a numerical keypad. By reporting which stimulus side they perceived as nearest, subjects implicitly indicated whether they perceived a slanted rectangle or a slanted trapezoid.

When viewing a slant rivalry stimulus, subjects report perceiving spontaneous alternations of their percept. In addition, they are able to voluntarily control their perception as well (van Ee et al. 2002, 2005b). To obtain equal sized data sets for each of both perceptual conditions, we used this ability to voluntarily control perception of the ambiguous stimulus. Thus, subjects were instructed to hold 
one of both possible perceived surfaces when viewing the bistable stimulus before the experiment started.

One experimental block consisted of a total of 16 trials, of which 8 were ambiguous stimuli conditions and the other 8 were unambiguous stimuli conditions. Both conditions were counter balanced for saccade direction and trials were randomized for conditions and saccade directions. Each experimental session contained three to four experimental blocks run consecutively. A calibration was performed, at the start of each block, i.e. every $5 \mathrm{~min}$.

\section{Data analysis}

Raw data provided by the Eyelink system was analyzed off line. We used a custom calibration procedure specifically designed to calibrate the raw data. This procedure consisted of a weighted least square error fit with a third order polynomial [35 measurement points, BFGS minimization technique (Press 1993)] (van Beers 2007). Offset correction was performed offline for each trial using the data obtained with the first fixation dot. On the calibrated data, saccade detection was performed based on version (mean gaze angle of left and right eye) velocity. A saccade was detected when the version velocity was higher than $120 \%$ s. The end of the saccade was defined when version velocity dropped below $20 \%$. Only saccades with an amplitude of at least $7^{\circ}$ in the indicated direction were analyzed. After the saccade, a slower vergence movement was usually present, vergence calculated as right eye gaze data-left eye gaze data. This was assumed to be part of the initial preprogrammed vergence. We defined the preprogrammed vergence offset when vergence velocity was minimum within a timeframe of $100 \mathrm{~ms}$ after saccade offset and $5 \% \mathrm{~s}$ at the most. We used the data at the point of minimum vergence velocity for further analysis. As we were interested in the vergence difference between the onset and offset of the saccade, we present the data relative to the gaze direction before onset of the saccade. Vergence data were averaged [three nearest neighbor average (24 ms interval)] to remove high-frequency noise.

\section{Subjects}

Subjects ( 6 females and 9 males aged between 18 and 30) had normal or corrected to normal vision. Before taking part in the experiment, it was checked whether a subject was able to comply with the instructions of the experiment. Not all potential subjects could perceive the trapezoidal interpretation of the stimulus within the timeframe of the experiment $(2 \mathrm{~s})$, when disparity and perspective foreshortening were in conflict. These subjects either had poor stereovision or needed more than $2 \mathrm{~s}$ to elicit a voluntary controlled flip. In total eight subjects were excluded from further participation, because it would have been impossible to measure the effect of depth perception on vergence.

\section{Results}

Saccades had normal main sequence characteristics (amplitudes of about $9^{\circ}$, durations of about $56 \mathrm{~ms}$, maximum velocities of about $310 \%$ s) (Collewijn et al. 1988). Most saccades reached an amplitude of $80-100 \%$ of the amplitude defined by the width of the stimulus. Sometimes a correction saccade was made after about $150 \mathrm{~ms}$. Only a small fraction of saccades (less than 2\%) was in the opposite direction as indicated by the monocular arrow presented.

\section{Experiment 1: Contribution of perception of depth} to vergence

To investigate whether perceived surface orientation contributes to vergence, we analyzed the data obtained during the presentation of the ambiguous stimulus. As mentioned in Sect. 2.3, we asked subjects to hold one of both possible orientations. In $52 \%$ of the 659 trials subjects reported perceiving a slanted rectangle and the slanted trapezoid was perceived in $48 \%$ of the trials. In $88 \%$ of all trials the percept was in agreement with the instruction given before the experiment started. The data of trials in which they failed (due to spontaneous or no (controlled) flips) are not included in the data presented here, but were not significantly different from the trials in which subjects were successful in obliging to the instruction.

\section{Predictions}

While the cues remained constant, perceived orientations alternated. Thus, we predict that if perception contributes to vergence, vergence should alternate accordingly. But, if not, the measured vergence changes should be independent of perceived slant orientation. Because we asked subjects to report only the sign of the orientation (and not the magnitude) of perceived slant, we can only predict the direction of the vergence changes, as illustrated in Fig. 4a. When subjects perceived the trapezoid (disparity-based percept), the saccades were directed towards the near side of the perceived surface, whereas saccades were directed towards the far side when subjects perceived the rectangle (perspective-based percept). Consequently, the vergence changes predicted by perception are in the converging direction (blue area) when subjects perceived a trapezoid and in the diverging direction (red area) when they perceived the rectangle. The upper and lower limits of the predicted vergence regions in Fig. 4a are defined by the vergence based on a zero depth change (grey line) and a vergence change based on the maximum slant defined by 


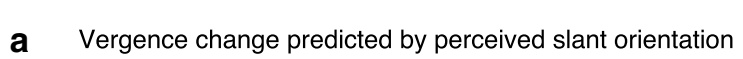

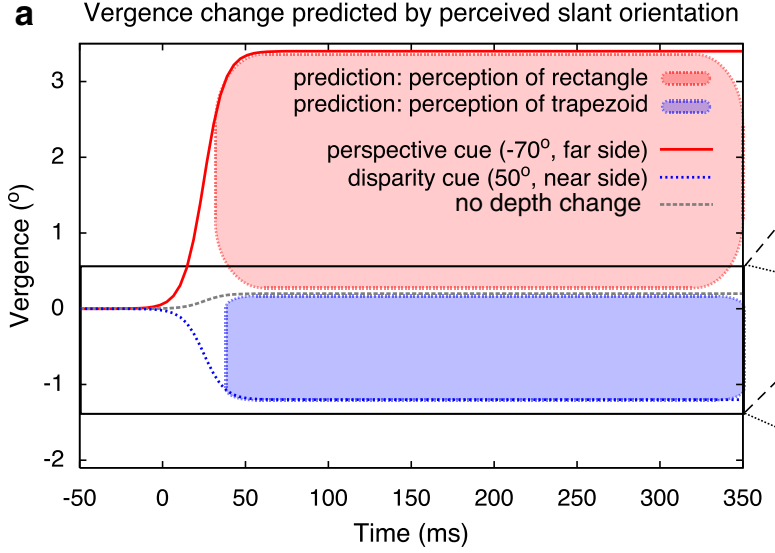

Fig. 4 a Predicted vergence changes based on perceived surface slant orientation. The transitions between vergence at saccade onset $(t=0 \mathrm{~ms})$ and at saccade offset are described using a sigmoid function. The upper and lower limits of the predicted vergence regions are based on the vergence step corresponding with the depth defined by a single cue. The box indicates the range of the detailed graphs in $\mathbf{b}$. b Vergence changes based on perceived surface slant orientation of subject $S 6$ (top) and subject $S 1$ (bottom). Mean traces for each condition with SE of

either depth cue: disparity (perception of trapezoid, blue line) or perspective (perception of rectangle, red line).

\section{Results}

Figure $4 \mathrm{~b}$ shows representative vergence traces from the "perception of trapezoid" and "perception of rectangle" conditions. These vergence traces are means of 25 trials at the least, with standard error (SE) displayed at every fourth data point. Saccade onset $(t=0 \mathrm{~ms})$ has been used to align the individual vergence traces for calculating the mean. Saccade offset occurred at $t=55 \mathrm{~ms}$ for S1 and at $t=46 \mathrm{~ms}$ for S6, which is in accordance with main sequence characteristics. Figure $4 \mathrm{~b}$ shows that at this point in time, the preprogrammed vergence movement has not ceased. Preprogrammed vergence offset was determined for individual traces (see Sect. 2.4) and cannot be pinpointed exactly in these graphs. Preprogrammed vergence offset typically occurred at $t=120 \mathrm{~ms}( \pm 30 \mathrm{~ms})$, at which point the vergence velocity (mean of all subjects) was $0.2 \%$ $\left(\mathrm{SD}=0.15^{\circ} \mathrm{s}\right)$. The diverging movement present in the onset of the saccades is commonly observed in most conjugate saccades and caused by temporal/nasal eye movement differences (Collewijn et al. 1988).

As Fig. $4 \mathrm{~b}$ shows, the vergence changed in the converging direction for both perceptual conditions. Moreover, all subjects showed converging responses. Thus, if subjects perceived a slanted trapezoid, vergence was in the same direction as the perceived orientation of the surface. And, if they perceived a slanted rectangle, the direction of vergence was opposite to the perceived orientation of the surface. This indicates that the contribution of perceived orientation to vergence, if any, is small. When comparing the data traces with the predictions in Fig. 4b, it is clear that the measured vergence changes are close to the value predicted by the disparity cue (blue dotted line). This result suggests that the largest contribution to vergence is related to the disparity cue.

To investigate the magnitude of the effect of perceived orientation on vergence, we further analyzed the data of all subjects, starting with selecting the end of the preprogrammed vergence movement as described in Sect. 2.4. We then normalized the data, using the calculated prediction based on the disparity cue, thereby effectively removing the variability caused by the interocular distance. ${ }^{2}$ These normalized data are presented in Fig. 5a. Because these data show individual biases (e.g. S4), we used the difference between the two conditions tested for calculations on group data (see Fig. 5b). The data of leftward (bottom) and rightward (top) saccades are shown separately, because individual biases varied across these conditions (e.g. for S4). ANOVA statistics on individual and group data showed that there was no significant difference between the two investigated conditions (except for S1 leftward saccade, see Fig. 5 caption), thereby rejecting the hypothesis that perceived orientation influences vergence. Thus, vergence is independent of perceived orientation.

\footnotetext{
${ }^{2}$ We used the prediction based on the disparity cue to define deviations, because a large part of the vergence change in the data of most subjects (except S4 rightward saccade) could be attributed to the disparity cue.
} 


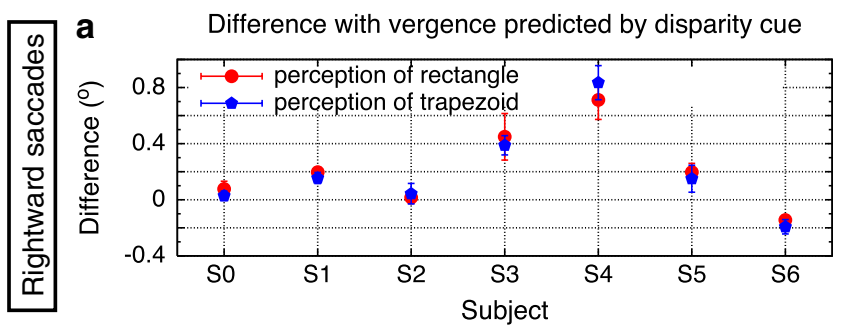

b Vergence relative to perception of trapezoid condition
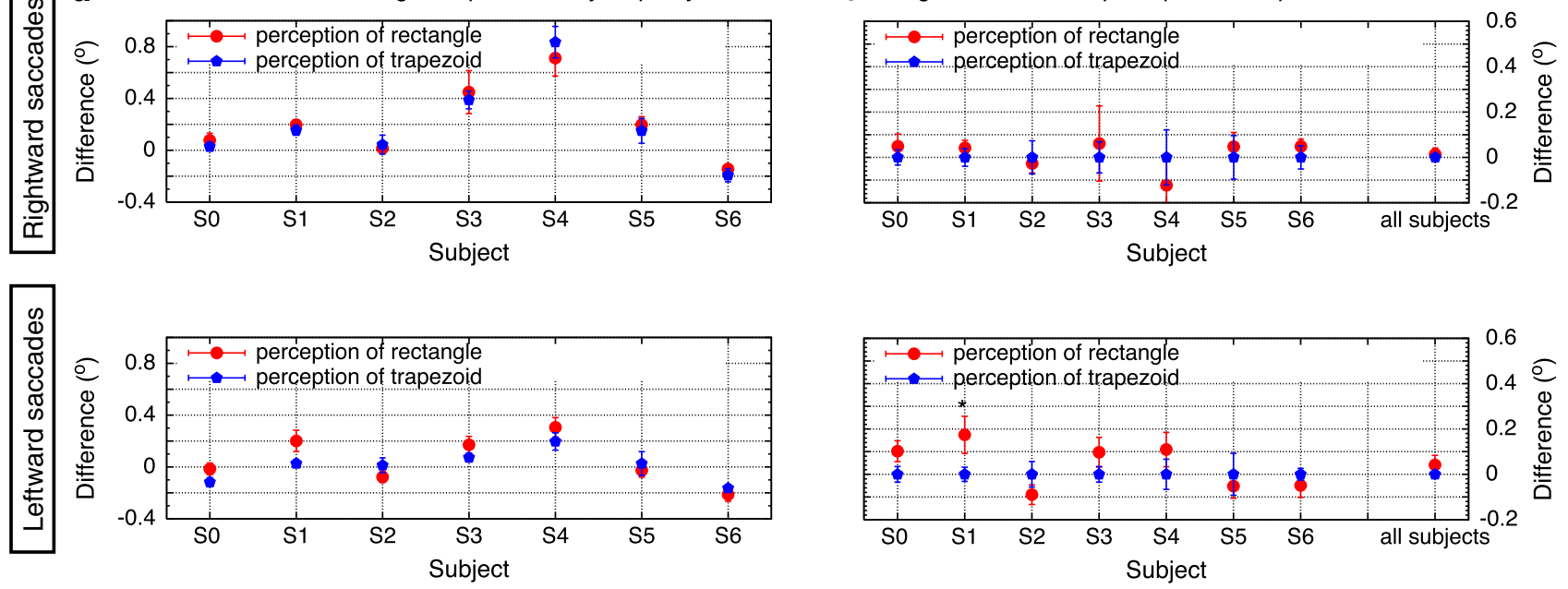

Fig. 5 a Vergence (mean and SE selected as described in Sect. 2.4) relative to predicted vergence based on the disparity cue $(0$ on vertical axis) from leftward saccade trials (bottom) and rightward saccade trials (top). These data show that (1) there are individual biases (e.g. S4), (2) the "perception of rectangle" (red circle) is not less convergent than the "perception of trapezoid" (blue pentagon) condition for all subjects (e.g. $S 2, S 5$ ) and both directions (e.g. $S 4, S 6$ ), rebutting the predictions

Experiment 2: Contribution of depth cues other than disparity to vergence

The results from experiment 1 show that perceived orientation does not influence vergence. Still, as stated in the introduction, cues other than disparity may contribute to vergence. We investigated this hypothesis by analyzing the data from the ambiguous stimulus and the unambiguous stimulus presentations.

\section{Predictions}

For both conditions the disparity defined slant was identical. In the unambiguous stimulus, the orientation of slant angle defined by perspective foreshortening was identical to the one defined by disparity, whereas it was opposite in the ambiguous stimulus. Thus, if perspective contributes to vergence, we predict that the vergence change of the ambiguous condition should be less convergent than that of the unambiguous condition. But, if not, vergence should be independent of the stimulus presented. Predictions of vergence changes based on slant cues are illustrated in Fig. 6a. For both conditions, the disparity cue signaled a slant of $50^{\circ}$ in magnitude and as saccades were directed towards the crossed disparity side of the stimulus, the corresponding predicted vergence change converges (blue dotted line). The perspective cue signaled a slant of $70^{\circ}$ in magnitude in both conditions. In the unambiguous condition, the vergence change predicted by perspective converges (green

based on perception. b Vergence (mean and SE selected as described in Sect. 2.4) relative to "perception of a trapezoid" condition ( 0 on vertical axis). On the right side of these graphs the mean (and SE) of all subjects is shown. There is no systematic trend visible across subjects. All differences are nonsignificant except for $S 1$, leftward saccade $(F=6.36, P<0.05)$

dashed line) and consequently, the predicted vergence change in the ambiguous condition diverges (red line).

\section{Results}

Figure $6 \mathrm{~b}$ shows representative traces of the unambiguous and ambiguous stimulus conditions. The vergence traces are means of 25 trials at the least with the SE displayed at every fourth data point. The characteristics of these vergence traces are similar to the traces in Fig. $4 \mathrm{~b}$. The vergence traces in Fig. $6 \mathrm{~b}$ both converge towards the predicted value based on the disparity cue, see Fig. 6a (blue dotted line). Thus, the contribution to vergence that can be related to disparity is relatively large, consistent with the results described before.

We further analyzed the data to investigate whether there was a significant effect of the perspective cue on vergence, using the same method as described in the previous subsection. Figure 7a shows the normalized data, whereas Fig. 7b displays the data from the ambiguous condition relative to the data from the unambiguous condition including the group means. Leftward (bottom) and rightward (top) saccades are shown separately. ANOVA statistics on group and individual data show that there is a significant difference between the ambiguous and unambiguous condition. The statistics for group data are: leftward saccades (lw) (difference $\left.=0.14^{\circ}, F=13.04, P<0.05\right)$ and rightward saccades (rw) (difference $=0.16^{\circ}, F=62.89, P<0.001$ ). And for individual subjects: $\mathrm{S} 0 \mathrm{lw}(F=11.29, P<0.01)$, S1 lw 


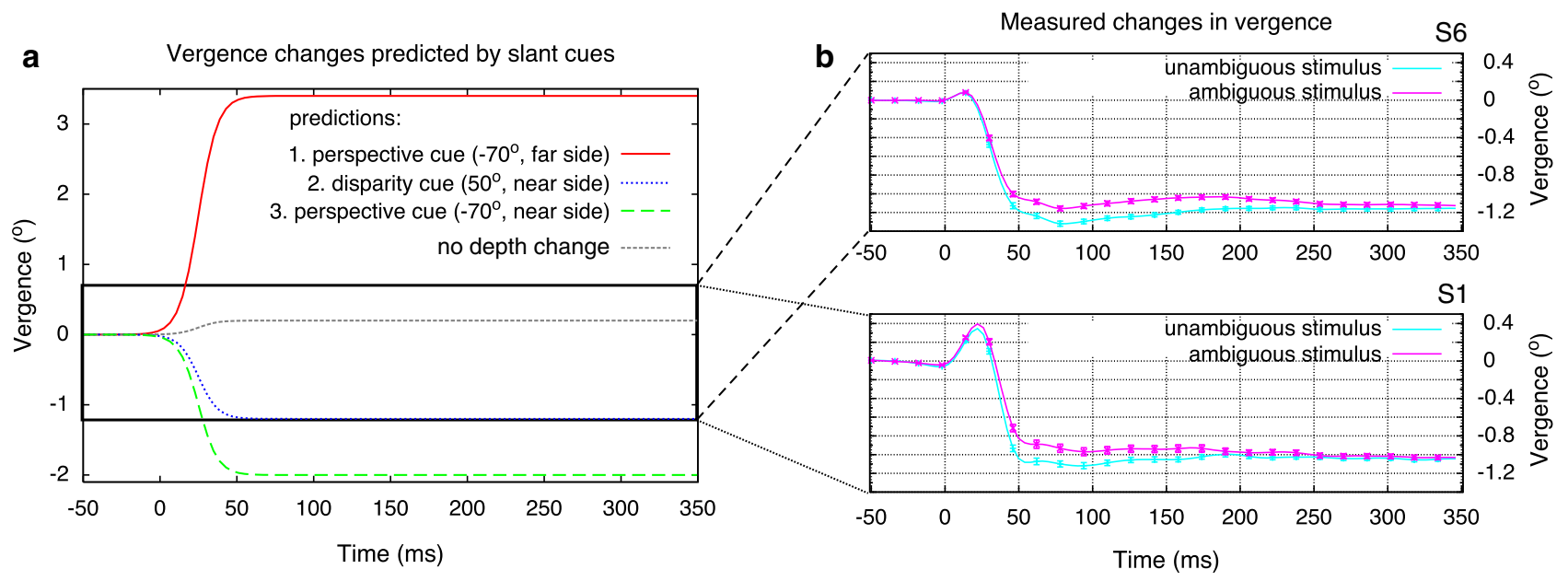

Fig. 6 a Predicted vergence changes based on the slant cues of the stimulus. The predicted values are based on the depth defined by a single cue. Details similar as in Fig. 4. b Vergence changes from ambiguous and unambiguous stimulus trials of subject $S 6$ (top) and subject $S 1$ (bottom). Mean traces of each condition with SE of every fourth

data point are displayed. Saccade offset occurred at $56 \mathrm{~ms}$ for $S 6$ and $46 \mathrm{~ms}$ for $S 1$ (saccade onset at $t=0 \mathrm{~ms}$ ). These data show that the vergence step of the ambiguous stimulus condition is less convergent than the vergence step of the unambiguous stimulus condition for both subjects

$(F=14.50, P<0.001)$ and $\mathrm{rw}(F=4.04, P<0.05), \mathrm{S} 2 \mathrm{lw}$ $(F=25.22, P<0.0001)$ and rw $(F=24.14, P<0.0001), \mathrm{S} 4$ lw $(F=24.84, P<0.0001)$, S6 lw $(F=5.22, P<0.05)$ and rw $(F=11.02, P<0.01)$.

Thus, perspective, or signals related to it, significantly contributed to vergence.

\section{Discussion}

Our findings show that depth cues rather than depth perception itself contribute to vergence accompanying saccadic movements. Perspective being congruent or incongruent with disparity caused a difference of about $14 \%$ in vergence changes predicted by disparity alone. Although our results show that vergence remained constant in the ambiguous stimulus condition, subjects perceived alternations of surface orientation. Collectively, these results show that perspective and disparity are each weighted differently for perception and vergence.

\section{Binocular studies}

Our result on the influence of perception on vergence refines the conclusions of previous studies relating perception and vergence (Sheliga and Miles 2003; Both et al. 2003). These studies investigated the influence of perception on vergence under binocular viewing conditions, similar as in our study. However, contributions of perception of depth to vergence could not be dissociated from those of depth-inducing cues. This dissociation was possible in our study. We found that, perspective contributes to vergence,

independent of its perceptual effect. This conclusion suggests that vertical disparities (Sheliga and Miles 2003), global horizontal disparities (Both et al. 2003) and perspective (present study) contribute to vergence similarly as local horizontal disparities do, albeit to a lesser extent.

\section{Monocular studies}

The perspective studies of Enright (1987a, b) show that vergence occurs corresponding to the depth implied by (linear) perspective under monocular viewing conditions. Enright concluded that the perceived depth contributed to vergence. However, in light of the present study, his results show that depth cues contribute to vergence under monocular viewing conditions as well. Moreover, without the dominantly contributing factor (disparity) being present, other depth cues become the main contributors to vergence.

The Necker cube study of Enright (1987a, b) and the ambiguous rotating sphere study of Ringach et al. (1996) seem to provide similar stimulus conditions as our experiment. The single depth cue used induced alternations in depth perception. In both studies corresponding alternations in vergence were measured and both authors conclude that depth perception induced vergence.

However, each depth cue used in these studies was inherently ambiguous. Thus, in these studies, the contribution of perception of depth to vergence could again not be dissociated from the contribution of the depth cues themselves. Taking the present study into consideration, there is evidence that their results reflect the alternations of the depth cue related signals and not those related to perception. In view of this conclusion, the fact that the vergence 


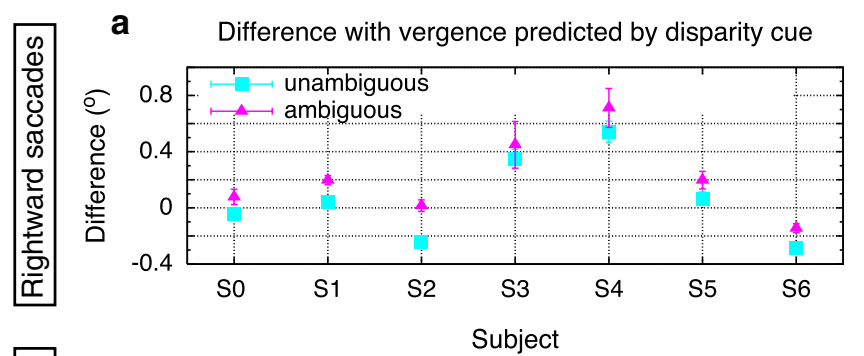

b Vergence relative to unambiguous condition
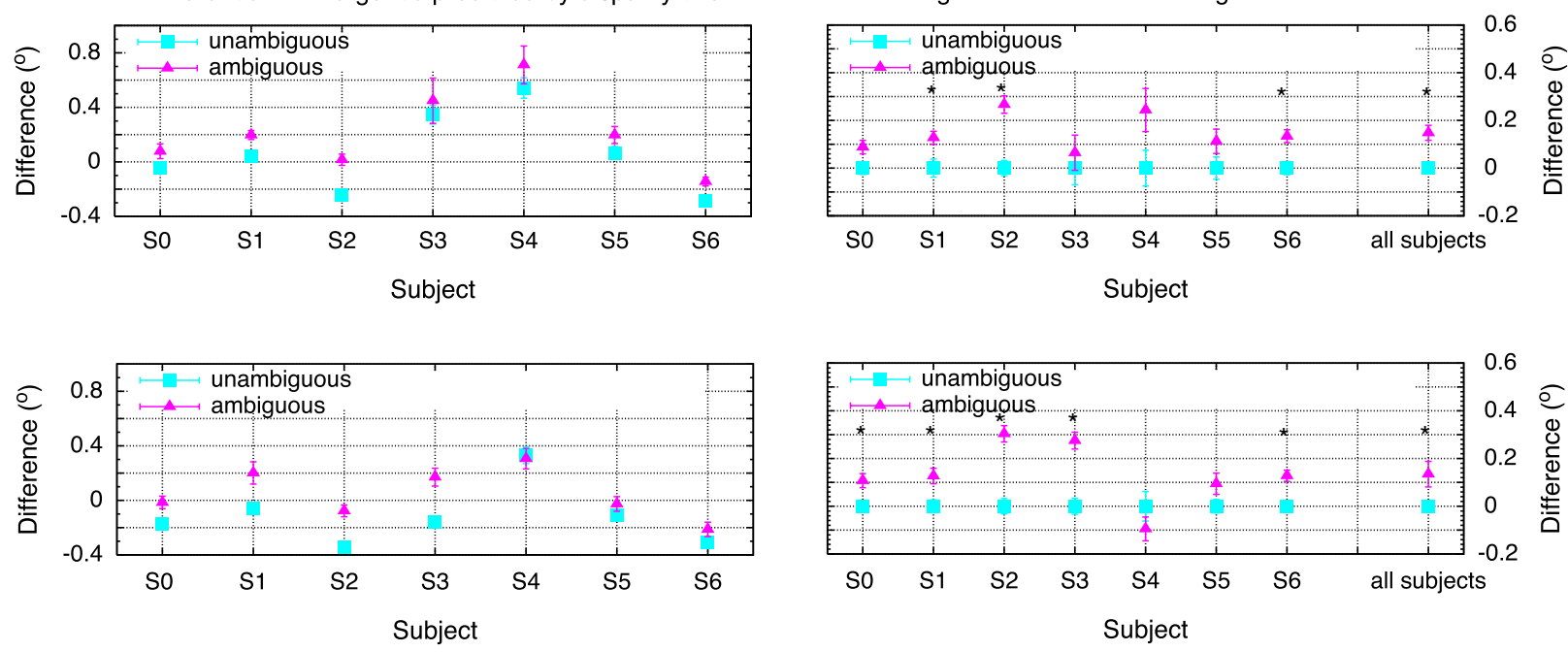

Fig. 7 a Vergence (mean and SE selected as described in Sect. 2.4) relative to predicted vergence based on the disparity cue $(0$ on vertical axis) from leftward saccade trials (bottom) and rightward saccade trials (top). Note that (1) there are some large individual biases, (2) that the ambiguous condition (purple triangle) is consistently less convergent than the unambiguous condition (cyan square), supporting the predic-

changes measured by Enright in the Necker cube experiment were much smaller than those in the linear perspective experiment may be explained by a degradation of the signals related to the depth cues due to the ambiguity herein.

\section{Influence of perception on other visual phenomena}

Our results indicate that vergence is influenced by lowlevel (sensory) processes and not by high-level (voluntary control) processes. Studies on bistability (van Ee et al. 2005b, a; Toppino 2003) show that high-level mechanisms can influence perception, whereas, as shown in the present study, they do not influence vergence. A recent study by Knapen and van Ee (2006) shows that slant adaptation is not influenced by perception or by voluntary control of perception, but is solely based on depth cues in the (slant rivalry) stimulus. Thus, both adaptation and vergence are influenced by low-level mechanisms, but not by high-level mechanisms.

\section{Different weighting of cues for vergence and perception}

To explain the current results and those of Enright (1987a, b) and Ringach et al. (1996), a single 3D surface representation used for perception and binocular eye movements as suggested by Ringach et al. (1996) is inappropriate. The perceptual states and the alternations thereof are not reflected in the vergence responses, suggesting that two separate representations are used for perception and eye movements. Moreover, the present results suggest that the

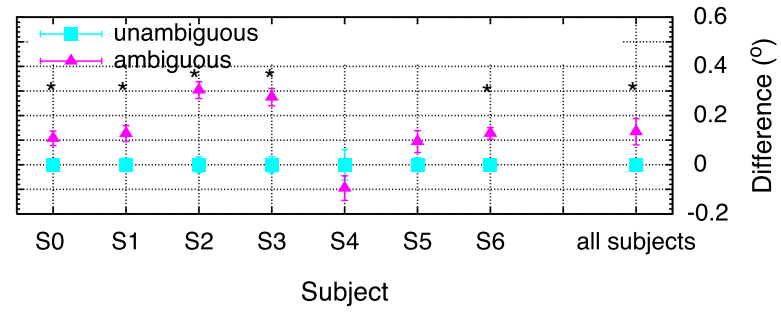

tions based on slant cues. b Vergence (mean and SE) relative to unambiguous condition. On the right side of these graphs the mean of all subjects is shown. The systematic trend visible between and across subjects is significant for the whole group (leftward saccades: $F=13.04, P<0.05$ and rightward: $F=62.89, P<0.001)$. All significant differences are denoted by a star (values are stated in text)

weights assigned to signals related to monocular and binocular depth cues are different for perception and vergence, resulting in either alternating or stable outcomes respectively, see Fig. 8. These outcomes may construct two separate $3 \mathrm{D}$ space representations one used for perception and

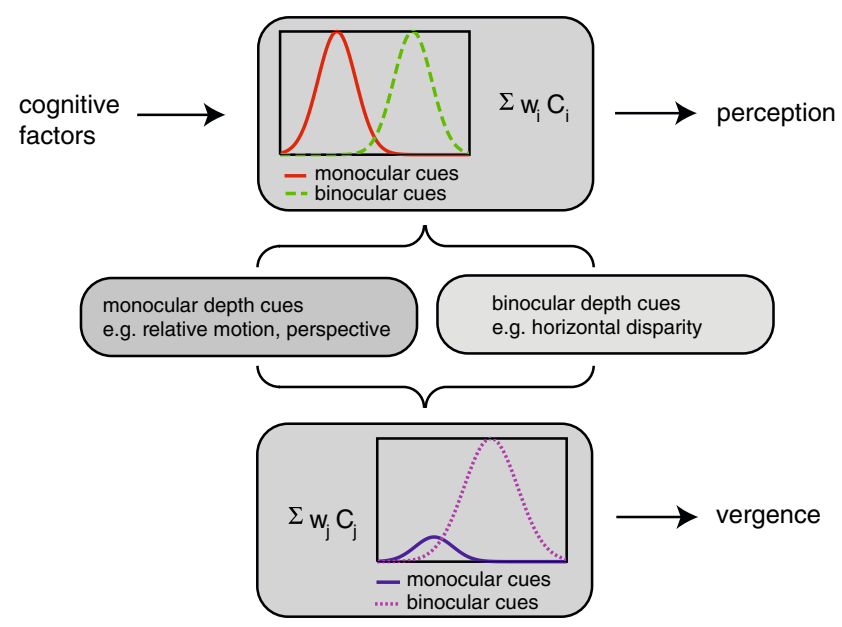

Fig. 8 Perception and vergence are based on separate processing streams. Both monocular cues (such as relative motion, linear perspective, blur and looming) and binocular cues [such as horizontal disparity (global and local) and global vertical disparity] are used for perception as well as for vergence. The weights assigned to the individual cues might be different when used for perception than when used for vergence, resulting in different outcomes. In the present study, for example, the ambiguous stimulus yields a bistable perception of surface slant, whereas it yields a stable vergence angle. Cognitive factors, such as voluntary control, do not seem to exert influence on vergence, but they do influence perception 
the other for vergence or, as an alternative, one 3D representation for perception whereas the signals for vergence are directly wired to the oculomotor system. However, in the latter situation the monocular signals must still be disambiguated before the motor command can be given to reorient the eyes.

\section{Perception and action}

Our results constitute another example of a dissociation between perception and action systems as suggested by Goodale and Milner (1992). Since then evidence supporting a dissociation has been accumulating, for a review see Goodale and Westwood (2004), Carey (2001) and Bruno (2001). Haffenden and Goodale (2000) and Haffenden et al. (2001) have shown that there are conditions in which perception has no influence on action, whereas other studies (Erkelens and Collewijn 1985; Masson et al. 1997; Schreiber et al. 2001; Banks et al. 2001; van Ee and van Dam 2003) have shown that conditions exist in which eye movements have no influence on visual perception. On the other hand, some studies conclude that action is influenced by perception (Franz et al. 2000, 2001; Franz 2001; Smeets et al. 2002; Lopez-Moliner et al. 2003; de Grave et al. 2006a, b; Bernardis et al. 2005; Knox and Bruno 2007). As we have shown all depth cues are used by the vergence system. The use of pictorial depth cues by other motor systems could perhaps explain this incongruence.

\section{Conclusion}

Our findings show that depth cues rather than perceived depth govern vergence that accompanies saccades. Perspective being congruent or incongruent with disparity caused a $14 \%$ difference in vergence change. These results combined with other studies show that in addition to local horizontal disparity, also global disparity, relative motion and perspective are used as input by the vergence system. Furthermore, our findings suggest that monocular and binocular cues are weighted differently for perception and vergence.

Acknowledgments The authors like to thank P. Schiphorst and H. Kolijn for their technical assistance and the subjects for their participation in the experiment. This project was supported by grants from the Netherlands Organization for Scientific Research (NWO) and the National Academy of Science (KNAW) awarded to RVE.

\section{References}

Allison RS, Howard IP, Rogers BJ, Bridge H (1998) Temporal aspects of slant and inclination perception. Perception 27(11):1287-1304

Banks MS, Hooge IT, Backus BT (2001) Perceiving slant about a horizontal axis from stereopsis. J Vis 1(2):55-79
Bernardis P, Knox P, Bruno N (2005) How does action resist visual illusion? Uncorrected oculomotor information does not account for accurate pointing in peripersonal space. Exp Brain Res 162(2):133-144

Both MH, van Ee R, Erkelens CJ (2003) Perceived slant from Werner's illusion affects binocular saccadic eye movements. J Vis 3(11):685-697

Bruno N (2001) When does action resist visual illusions? Trends Cogn Sci 5(9):379-382

Carey D (2001) Do action systems resist visual illusions? Trends Cogn Sci 5(3):109-113

Collewijn H, Erkelens CJ, Steinman RM (1988) Binocular co-ordination of human horizontal saccadic eye movements. J Physiol 404:157-182

de Grave DDJ, Franz VH, Gegenfurtner KR (2006a) The influence of the brentano illusion on eye and hand movements. J Vis 6(7):727-738

de Grave DDJ, Smeets JBJ, Brenner E (2006b) Why are saccades influenced by the brentano illusion? Exp Brain Res 175(1):177-182

Enright JT (1984) Changes in vergence mediated by saccades. J Physiol 350:9-31

Enright JT (1987a) Art and the oculomotor system: perspective illustrations evoke vergence changes. Perception 16(6):731-746

Enright JT (1987b) Perspective vergence: oculomotor responses to line drawings. Vision Res 27(9):1513-1526

Erkelens CJ, Collewijn H (1985) Eye movements and stereopsis during dichoptic viewing of moving random-dot stereograms. Vision Res 25(11):1689-1700

Erkelens CJ, Steinman RM, Collewijn H (1989) Ocular vergence under natural conditions. ii. Gaze shifts between real targets differing in distance and direction. Proc R Soc Lond B Biol Sci 236(1285):441-465

Franz V (2001) Action does not resist visual illusions. Trends Cogn Sci 5(11):457-459

Franz VH, Gegenfurtner KR, Bulthoff HH, Fahle M (2000) Grasping visual illusions: no evidence for a dissociation between perception and action. Psychol Sci 11(1):20-25

Franz VH, Fahle M, Bulthoff HH, Gegenfurtner KR (2001) Effects of visual illusions on grasping. J Exp Psychol Hum Percept Perform 27(5):1124-1144

Gillam BJ (1968) Perception of slant when perspective and stereopsis conflict: experiments with aniseikonic lenses. J Exp Psychol 78(2):299-305

Gillam B, Ryan C (1992) Perspective, orientation disparity, and anisotropy in stereoscopic slant perception. Perception 21(4):427-439

Gillam BJ, Cook ML (2001) Perspective based on stereopsis and occlusion. Psychol Sci 12(5):424-429

Goodale MA, Milner AD (1992) Separate visual pathways for perception and action. Trends Neurosci 15(1):20-25

Goodale MA, Westwood DA (2004) An evolving view of duplex vision: separate but interacting cortical pathways for perception and action. Curr Opin Neurobiol 14(2):203-211

Haffenden AM, Goodale MA (2000) Independent effects of pictorial displays on perception and action. Vision Res 40(10-12):15971607

Haffenden AM, Schiff KC, Goodale MA (2001) The dissociation between perception and action in the ebbinghaus illusion: nonillusory effects of pictorial cues on grasp. Curr Biol 11(3):177-181

Hoffmann J, Sebald A (2007) Eye vergence is susceptible to the hollow-face illusion. Perception 36(3):461-470

Howard IP (2002) Seeing in depth, Basic Mechanisms. I. Porteous, vol 1

Knapen T, van Ee R (2006) Slant perception, and its voluntary control, do not govern the slant aftereffect: multiple slant signals adapt independently. Vision Res 46(20):3381-3392

Knox P, Bruno N (2007) When does action resist visual illusion? The effect of Muller-Lyer stimuli on reflexive and voluntary saccades. Exp Brain Res 181(2):277-287 
Lopez-Moliner J, Smeets JBJ, Brenner E (2003) Similar effects of a motion-in-depth illusion on manual tracking and perceptual judgements. Exp Brain Res 151(4):553-556

Masson GS, Busettini C, Miles FA (1997) Vergence eye movements in response to binocular disparity without depth perception. Nature 389(6648):283-286

Press W (1993) Numerical recipes for C. Cambridge University Press, London

Ringach DL, Hawken MJ, Shapley R (1996) Binocular eye movements caused by the perception of three-dimensional structure from motion. Vision Res 36(10):1479-1492

Schreiber K, Crawford JD, Fetter M, Tweed D (2001) The motor side of depth vision. Nature 410(6830):819-822

Sheliga BM, Miles FA (2003) Perception can influence the vergence responses associated with open-loop gaze shifts in $3 \mathrm{~d}$. J Vis 3(11):654-676

Smeets JBJ, Brenner E, de Grave DDJ, Cuijpers RH (2002) Illusions in action: consequences of inconsistent processing of spatial attributes. Exp Brain Res 147(2):135-144
Toppino TC (2003) Reversible-figure perception: mechanisms of intentional control. Percept Psychophys 65(8):1285-1295

van Beers RJ (2007) The sources of variability in saccadic eye movements. J Neurosci 27 (in press). doi:10.1523/JNEUROSCI.231107.2007

van Ee R, van Dam LCJ (2003) The influence of cyclovergence on unconstrained stereoscopic matching. Vision Res 43(3):307319

van Ee R, Krumina G, Pont S, van der Ven S (2005a) Voluntarily controlled bi-stable slant perception of real and photographed surfaces. Proc Biol Sci 272(1559): 141-148

van Ee R, van Dam LCJ, Brouwer GJ (2005b) Voluntary control and the dynamics of perceptual bi-stability. Vision Res 45(1):41-55

van Ee R, van Dam LCJ, Erkelens CJ (2002) Bi-stability in perceived slant when binocular disparity and monocular perspective specify different slants. J Vis 2(9):597-607 